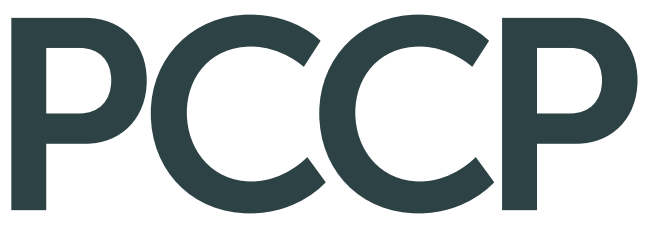

Physical Chemistry Chemical Physics rsc.li/pccp

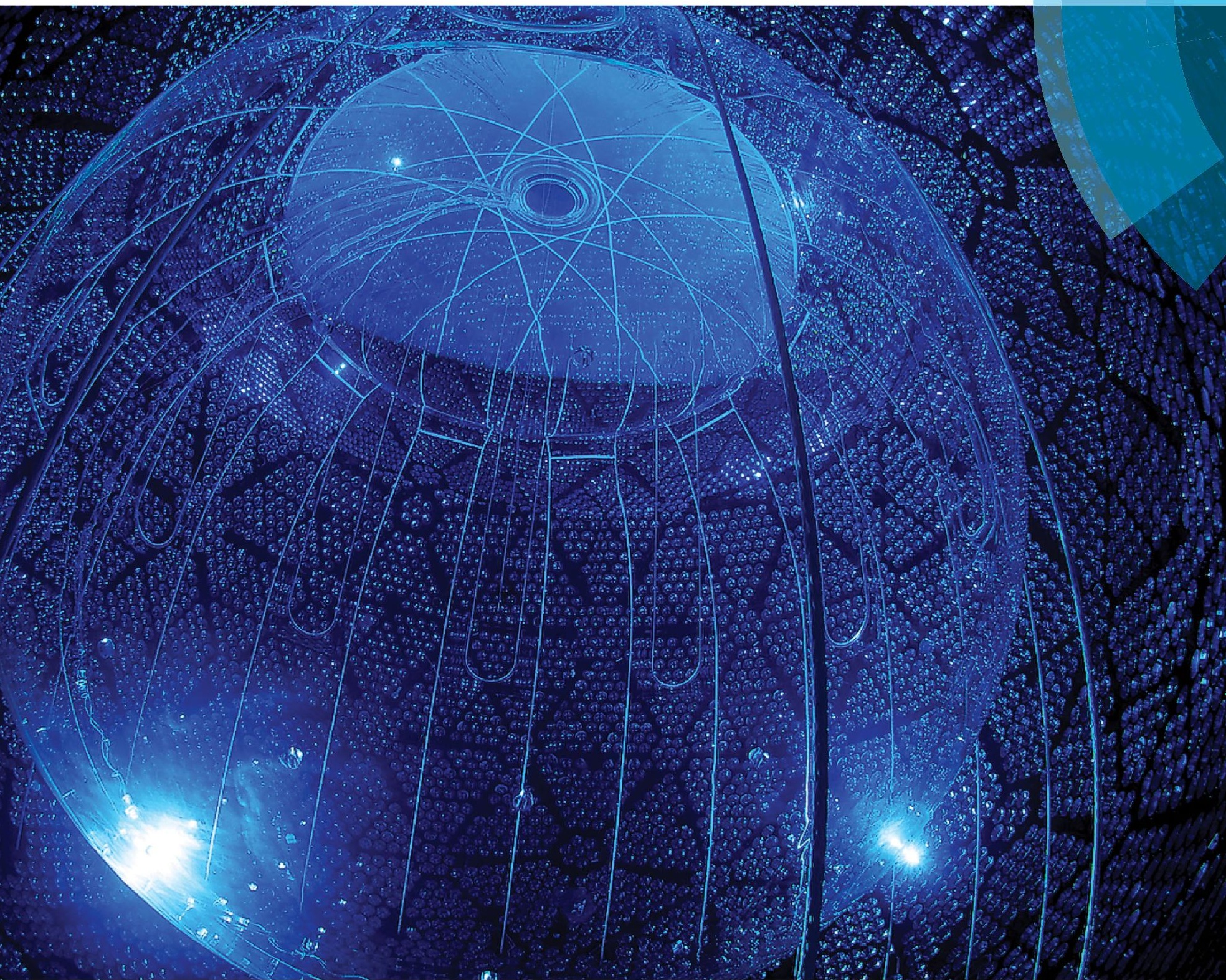

ISSN 1463-9076

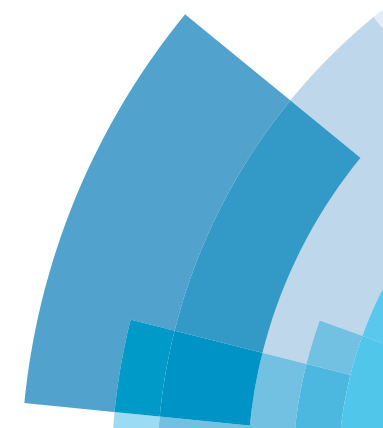




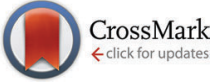

Cite this: Phys. Chem. Chem. Phys., 2017, 19, 73

Received 31st August 2016, Accepted 20th November 2016

DOI: $10.1039 / c 6 c p 06015 c$

www.rsc.org/pccp

\title{
Determination of the thermal, oxidative and photochemical degradation rates of scintillator liquid by fluorescence EEM spectroscopy $\dagger$
}

\begin{abstract}
N. L. P. Andrews, J. Z. Fan, R. L. Forward, M. C. Chen and H.-P. Loock*
The thermal, oxidative and photochemical stability of the scintillator liquid proposed for the SNO+ experiment has been tested experimentally using accelerated aging methods. The stability of the scintillator constituents was determined through fluorescence excitation emission matrix (EEM) spectroscopy and absorption spectroscopy, using parallel factor analysis (PARAFAC) as an multivariate analysis tool. By exposing the scintillator liquid to a well-known photon flux at $365 \mathrm{~nm}$ and by measuring the decay rate of the fluorescence shifters and the formation rate of their photochemical degradation products, we can place an upper limit on the acceptable photon flux as $1.38 \pm 0.09 \times 10^{-11}$ photon mol L $\mathrm{L}^{-1}$. Similarly, the oxidative stability of the scintillator liquid was determined by exposure to air at several elevated temperatures. Through measurement of the corresponding activation energy it was determined that the average oxygen concentration would have to be kept below 4.3-7.1 $\mathrm{ppb}_{\mathrm{w}}$ (headspace partial pressure below 24 ppm $_{v}$ ). On the other hand, the thermal stability of the scintillator cocktail in the absence of light and oxygen was remarkable and poses no concern to the SNO+ experiment.
\end{abstract}

\section{Introduction}

The Sudbury Neutrino Observatory, SNO, was built at the 6800-foot level in the Vale Creighton mine near Sudbury, Ontario. ${ }^{1}$ Its successor, the upcoming SNO+ project, uses an organic solvent as the liquid scintillator (replacing the heavy water that was in $\mathrm{SNO}$ ) to detect neutrinos. The SNO+ project will study solar neutrinos, reactor and geo antineutrinos, supernova neutrinos, and especially neutrinoless double beta decay $(0 \nu \beta \beta){ }^{2}$ If neutrinos are Majorana particles (as opposed to Dirac particles), and thereby their own antiparticles, doublebeta decay events may occur without neutrino emission. To observe $0 \nu \beta \beta$ experimentally, a double-beta decaying isotope, ${ }^{130} \mathrm{Te}$, will be used. The search for the $\beta \beta$-decay process can be achieved by detecting the light emission generated by the $\beta$-particles from decaying ${ }^{130}$ Te nuclei as they interact with the scintillator cocktail. The scintillator is an organic solvent mixture, which also contains the dissolved or emulsified tellurium. Several thousand photomultiplier tubes (PMTs) are dedicated to detecting the emission in the visible region of the spectrum and the liquid scintillator cocktail therefore contains several fluorescence shifters, i.e. dyes that absorb the primary UV emission and fluoresce at longer wavelengths. In addition

Department of Chemistry, Queen's University, Kingston, ON, K7L 3N6, Canada. E-mail: hploock@queensu.ca

$\dagger$ Electronic supplementary information (ESI) available. See DOI: 10.1039/c6cp06015c to the primary fluorophore, which also acts as a solvent, the scintillator cocktail contains strongly fluorescent compounds that through absorption of the primary fluorescence and subsequent fluorescence emission at longer wavelength, shift the emission to the optimum wavelength for optimal light detection. Specifically, for the $\mathrm{SNO}+$ experiment, the scintillator cocktail under consideration comprises of a mixture of the primary fluorophore, linear alkylbenzene (LAB), a secondary fluorophore, 2,5-diphenyloxazole (PPO), and a tertiary fluorophore, 1,4-bis(2-methylstyryl)benzene (bis-MSB). Telluric acid, $\mathrm{Te}(\mathrm{OH})_{6}$, is not readily soluble in LAB and may either be encapsulated by micelles of dodecylbenzenesulfonic acid 2-propanamine, a surfactant, or may reacted with e.g. diols to form a soluble complex. The fluorescence shifters are selected such that the emission by LAB is absorbed by PPO and the PPO emission is absorbed by bis-MSB. The emission of PPO and bis-MSB falls into a window of high optical transparency of the primary fluorophore and solvent $\mathrm{LAB}$ as well as into a sensitive region of the photodetector response spectrum. Similar mixtures of fluorophores are also used in other neutrino detectors such as the Daya Bay Reactor Neutrino Experiment and the Reactor Experiment for Neutrino Oscillations (RENO). ${ }^{3,4}$ The acrylic vessel for the SNO+ experiment will hold approximately 780 tonnes of liquid scintillator loaded with around 3.9 tonnes of tellurium. Over 9500 PMTs surround the $\mathrm{SNO}+$ vessel to detect the light emission from the fluorescing species in solution. 
It is anticipated that $0 \nu \beta \beta$ is, at best, an extremely rare process producing only a few events per year, and the SNO+ experiment will have to be conducted for around seven years in a largely inert atmosphere at around $12{ }^{\circ} \mathrm{C}$. During this period, the scintillator cocktail may start to decompose, thereby reducing the overall quantum yield of the cocktail. This would be detrimental to the SNO+ experiment, since the stability of the detector light yield is important for determining the energy of detected neutrinos and especially critical for the double-beta decay search. Also, colored degradation products may reduce the transparency of the cocktail and thereby reduce the intensity of the detectable emission.

In this study we independently consider thermal decomposition, oxidative destruction, and photochemical degradation. For these three processes we obtain rate constants and associated activation energies. This allows us to estimate the tolerances that are acceptable to maintain a functioning scintillator liquid.

A replica of the scintillator cocktail, not containing tellurium, was artificially aged at several high temperatures either under inert atmosphere or with exposure to air. The scintillator cocktail was also exposed to UV (365 nm) light. At high temperatures these thermal and photochemical aging processes take several days rather than several years. Rate constants and activation energies were determined and the decay rate at ambient temperatures was estimated by extrapolation. Fluorescence excitation emission matrix spectroscopy (EEMS) was used to quantify the concentrations of fluorophores while absorption spectroscopy helped determine the product formation rates.

Fluorescence EEMS combined with a multivariate analysis method is commonly used to classify compounds such as foodstuff, ${ }^{5}$ environmental, ${ }^{6}$ and industrial liquids. ${ }^{7,8}$ EEMS is also a powerful but possibly underused technique uniquely suited to follow the kinetics of fluorescent compounds for example in estimating the remaining useful life of engine lubricants. ${ }^{7-9}$ By comparison to other analytical techniques such as gas chromatography (GC) and high-performance liquid chromatography (HPLC), EEMS is non-destructive, does not require sample preparation and can be used with a fiber probe for on-line analysis, i.e. it might be useful in at-line quality monitoring - not only of scintillation liquids. In EEMS the sample is excited at a large number of wavelengths and the entire emission spectrum is recorded at each excitation wavelength. This generates threedimensional fluorescence maps that allow the observation of all of the fluorescing compounds within the excitation boundaries. These second-order EEM spectra can be interpreted using a multivariate analysis technique such as parallel factor (PARAFAC) analysis to identify and quantify the spectroscopic components within the cocktail. In some cases, such as the present study, these PARAFAC components may be correlated to the fluorescent chemical compounds or compound classes. ${ }^{10,11}$

Our study is intended to guide the scintillator composition of the $\mathrm{SNO}+$ project, and allow us to specify the conditions that permit the scintillator to function throughout the several year duration of the SNO+ experiment. It may also serve as an example on the application of fluorescence EEM in determining chemical decay kinetics.

\section{Experimental section}

\section{Sample preparation}

Samples contained $2 \mathrm{~g} \mathrm{~L}^{-1}$ PPO (Top Grade, PerkinElmer), $15 \mathrm{mg} \mathrm{L}{ }^{-1}$ bis-MSB (Aldrich, Milwaukee, WI) all dissolved in LAB (Petresa Canada Inc.). The modified replicate cocktail was identical to that proposed for $\mathrm{SNO}+$ with the exception of the tellurium-containing micelles consisting of $\mathrm{Te}(\mathrm{OH})_{6}$ and surfactant. These compounds do not contribute or alter the fluorescence of the scintillator (Fig. 1).

\section{Aging experiments}

Thermal aging in air. The replica SNO+ cocktail $(200 \mathrm{~mL})$ was heated under reflux under a constant stream of breathing grade air (Praxair) for one to three days. The samples were heated to $397,426,444,455,466 \mathrm{~K}$ and the temperature was maintained for the entire duration of the experiment. $5 \mathrm{~mL}$ aliquots were withdrawn at logarithmic time intervals over the course of up to three days.

Thermal aging under nitrogen. In order to thermally degrade samples under an inert atmosphere, the replica SNO+ solution was degassed by bubbling nitrogen gas ( 4.8 nitrogen, PRAXAIR) for 24 hours. $^{12,13}$ The solution was then placed in a nitrogen glove bag where $4 \mathrm{~mL}$ of $\mathrm{SNO}+$ solution was added to ampules and temporarily capped. The ampules were subsequently evacuated using a vacuum pump and refilled with nitrogen. Evacuation and backfilling was repeated twice. The ampules were then sealed using a propane torch. To age the samples the ampules were placed in a recrystallization oven which held a steady temperature of $513 \pm 1 \mathrm{~K}$. The ampules were removed on a semi-logarithmic time scale, and, once cooled, they were used for subsequent analysis.

Photochemical aging. To remove oxygen the replica $\mathrm{SNO}+$ cocktail and a PPO solution ( $2 \mathrm{~g} \mathrm{~L}^{-1}$ PPO dissolved in LAB) were both degassed using a constant flow of high purity nitrogen gas (4.8 nitrogen, PRAXAIR) for over 48 hours prior to the UV aging experiment. The SNO+ cocktail was then exposed to UV light (Bondwand $365 \mathrm{~nm}$ UV curing lamp, Electrolite, USA), at room temperature $(298 \mathrm{~K})$. The photon flux through the sample was determined as $8.49 \pm 0.35$ photon $\mathrm{mol} \mathrm{s}^{-1}$ with a ferrioxalate actinometer using an established method by Hatchard et al. ${ }^{14}$

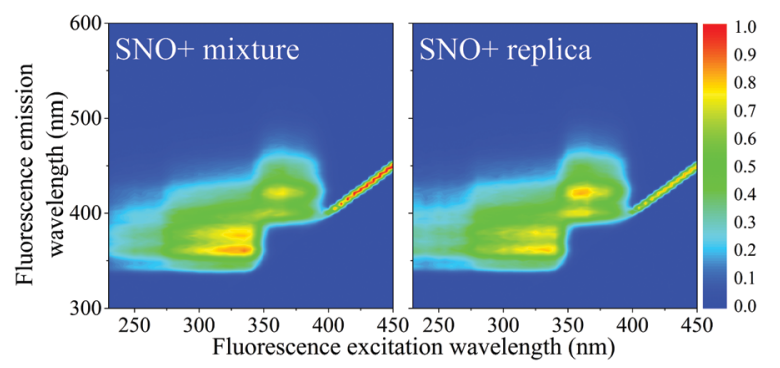

Fig. 1 Normalized EEM spectra of the original SNO+ cocktail (left: $15 \mathrm{mg} \mathrm{L}^{-1}$ bis-MSB, $2 \mathrm{~g} \mathrm{~L}^{-1} \mathrm{PPO}$, micelles of $\mathrm{Te}(\mathrm{OH})_{6}$ and surfactant, in $\mathrm{LAB}$ ) and of the replica SNO+ cocktail (right: $15 \mathrm{mg} \mathrm{L}^{-1}$ bis-MSB, $2 \mathrm{~g} \mathrm{~L}^{-1} \mathrm{PPO}$ in LAB). The colors from blue to red represent the normalized intensity of 0 to 1. 
This corresponds to a concentration of $[h \nu]=4.25 \pm 0.17 \times 10^{-7}$ photon $\mathrm{mol} \mathrm{L}^{-1}$. The power density of the lamp at $365 \mathrm{~nm}$ was determined to be $1.19 \pm 0.05 \mathrm{~mW} \mathrm{~cm}^{-2}$. The UV lamp was placed under the sample vessel, and the sample was exposed to UV light in 60 minute intervals. After each irradiation interval the sample remained in the dark for 30 minutes. The periodic irradiation ensured that the UV lamp would not overheat or burn out. The SNO+ cocktail was exposed to UV light for approximately 1 week. The entire setup was contained in a black box to minimize degradation by ambient light.

\section{Absorption measurements}

Absorption spectra were acquired for 91 of the 101 degraded scintillation solutions (Ocean Optics USB4000 spectrometer coupled to an Ocean Optics USB-ISS-UV-Vis light source). Since thermal aging under an inert atmosphere had no measurable effect, not all samples from this experiment were analysed. For each sample 10 spectra were averaged with an integration time of $60 \mathrm{~ms}$. The oxidized samples were placed in a $1 \mathrm{~mm}$ cuvette and were diluted with LAB.

\section{Excitation emission matrix spectroscopy}

A double-grating fluorescence spectrometer (Varian, Cary Eclipse) was used to collect EEM spectra of all 101 aged sample solutions. Approximately $3.5 \mathrm{~mL}$ of each sample was placed into a $10 \times 10 \times 45 \mathrm{~mm}$ quartz-fused cuvette. The spectra acquisition was performed in the dark to ensure minimal exposure to ambient light. A custom-built bifurcated fiber-optic cable bundle was coupled directly to the spectrometer. The design of the fiber probe is discussed in detail in a previously published paper. ${ }^{9}$ The custom probe was used instead of conventional right angle fluorescence collection, since it is less affected by the secondary absorption by the fluorophores, which is inherent in scintillation cocktails.

All EEM spectra for PARAFAC analysis were collected in an excitation range of $200-450 \mathrm{~nm}$ with an emission range of 300-600 nm. The excitation and emission wavelengths had step increments of $5 \mathrm{~nm}$ and $2 \mathrm{~nm}$, respectively. Both of the excitation and emission monochromator slits were set to $5 \mathrm{~nm}$ and autofilters were used to remove second-order diffraction light from exciting the sample. A scan rate of $1200 \mathrm{~nm}$ per minute was used for the fluorescence collection and each of the 101 spectra took approximately 28 minutes to collect.

\section{Parallel factor (PARAFAC) analysis}

All 101 EEM spectra were analyzed together using multivariate analysis. PARAFAC analysis was performed using the drEEM toolbox in Matlab ${ }^{\mathrm{TM}}$ to identify specific fluorescent compounds in the SNO+ cocktail. ${ }^{10,11}$ In contrast to most other multivariate methods PARAFAC can sometimes help identify specific fluorophores or classes of fluorophores. In our case of initially wellcharacterized samples, whose composition evolves with time, PARAFAC is a particularly useful technique. The PARAFAC analysis algorithm was applied 10 times to the data set, with random initialization, non-negativity constraints, and a convergence criterion of $1 \times 10^{-10}$. The spectra were also normalized before analysis to give equal weights to those samples having weak fluorescence.

The analysis of the complete data set of 101 EEM spectra gave an excellent fit to only three distinct components, as shown in Fig. 2, with a "percent explained" of $96.0 \%$ and a core consistency of $96.7 \%$, meaning that the components are largely orthogonal. Two of the components-F1 and F2-clearly relate to the two scintillation shifters-PPO and bis-MSB, respectively. ${ }^{10,11,15}$ (a)

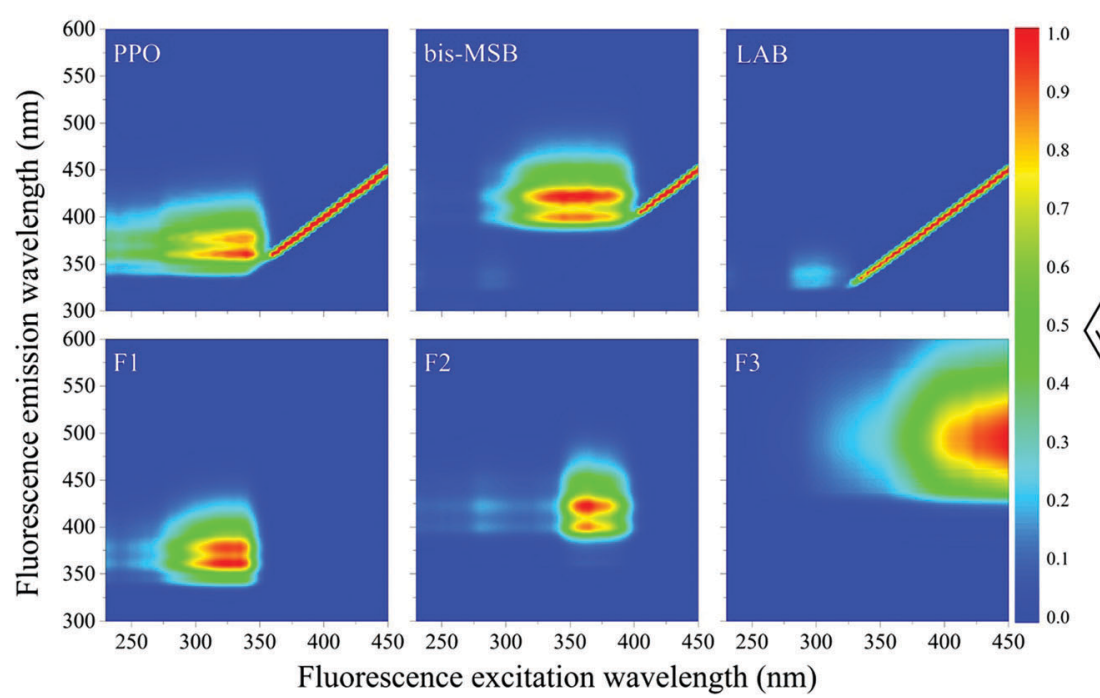

(b)<smiles></smiles>

(c)
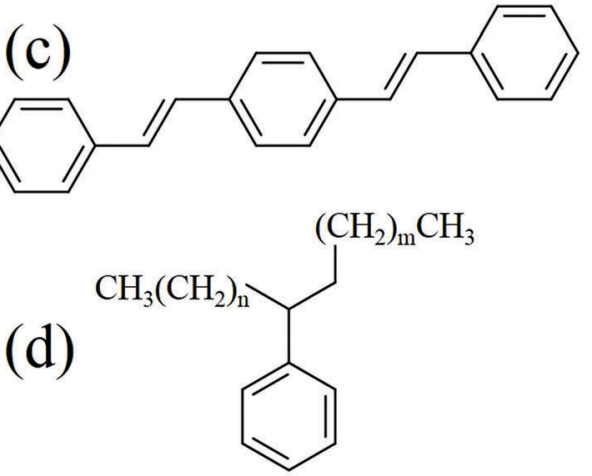

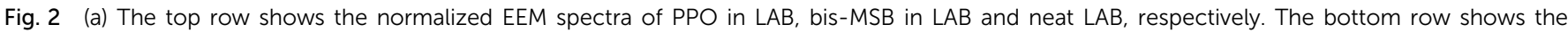

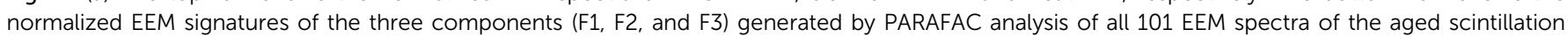
solutions. (b), (c), and (d) represent the structures of PPO, bis-MSB and LAB respectively, where $n+m=6-12$. 
Their EEM spectra were recorded independently in the solvent $\mathrm{LAB}$ and were very similar to those of the two components in Fig. 2. The differences between the spectra are attributed to inner filter effects. The third component F3, which is red-shifted from F1 and F2 is most likely attributable to dimers and oligomers formed as the $\mathrm{SNO}+$ scintillation solution is aged. The identity of these compounds is discussed below.

Notably, fluorescence of LAB is not observed. LAB exhibits an excitation maximum near $300 \mathrm{~nm}$ and emission at around $330 \mathrm{~nm}$. LAB fluorescence is not observed in the presence of PPO, since PPO absorbs the fluorescence of LAB. The LAB peak is faintly observed in the bis-MSB sample, however.

In addition, the absorption spectra (67 oxidized and 7 thermally aged in inert conditions) were analyzed using the same PARAFAC analysis to determine any increased absorption due to the formation of products from the degradation of the scintillator solution. The 17 absorption spectra of the photodegraded samples underwent a separate PARAFAC analysis. Since the drEEM toolbox requires a 3-dimensional data cube, the square root of the 2-D absorption spectra were multiplied by the square root of their transpose (decimated by 10) to generate a mock-EEM spectrum that could be analyzed. ${ }^{16}$ This ensured that the same number of components was present in both dimensions and that the magnitude of absorption matched that of the sample.

\section{Kinetic model}

The concentration of both fluorophores, bis-MSB and PPO, may be described with a simple kinetic model based on pseudo-first order reactions. As the scintillation solution is heated either with or without oxygen, or degraded using UV radiation, the fluorophore, $\mathrm{X}$, degrades into one or more products, such as an oxidation product, a dimer or higher oligomer. Also, it is likely that, when heated or photochemically excited, bis-MSB isomerizes from its trans- to cis-form. In the cis-configuration a reversible pericyclic reaction can occur where the cis-conjugated triene forms a cyclohexadiene, which in this case is a dihydrophenanthrene. ${ }^{17}$ With the addition of an oxidizer such as $\mathrm{O}_{2}$ the dihydrophenanthrene intermediate can form phenanthrene. ${ }^{18,19}$ PPO when heated in the presence of oxygen will form an ozonide. ${ }^{20}$ This will break the conjugation and thus reduce the fluorescence in the visible region. The ozonide can then further react to form ketones and aldehydes. ${ }^{21}$ In the presence of UV radiation PPO can undergo a photochemical rearrangement to form 3,5-diphenylisoxazole with 2,4-diphenyloxazole. ${ }^{22}$ The identity of the products is not of great relevance to this study, but their collective fluorescence and absorption properties are important. We model a generic decomposition of fluorophore $\mathrm{X}$ using the following reactions.

$$
\begin{gathered}
\mathrm{X}+\mathrm{O}_{2} \underset{\Delta}{\stackrel{k_{\mathrm{O}_{2}}}{\longrightarrow}} \mathrm{P}_{\mathrm{O}_{2}} \text { where } \mathrm{X}=\mathrm{PPO}, \text { bis-MSB } \\
\mathrm{X} \underset{\Delta}{\stackrel{k_{\mathrm{T}}}{\longrightarrow}} \mathrm{P}_{\mathrm{T}}
\end{gathered}
$$

$$
\mathrm{X}+h \nu \stackrel{k_{\mathrm{P}}}{\longrightarrow} \mathrm{P}_{\mathrm{P}}
$$

and if the product degrades further:

$$
\mathrm{P}_{\mathrm{O}_{2}}, \mathrm{P}_{\mathrm{T}}, \mathrm{P}_{\mathrm{P}} \stackrel{k_{\text {Product }}}{\longrightarrow} \mathrm{P}_{\mathrm{F}}
$$

The concentrations of the fluorophore, $\mathrm{X}$, are therefore governed by the rate equations:

$$
\begin{gathered}
-\frac{\mathrm{d}[\mathrm{X}]}{\mathrm{d} t}=k_{\mathrm{O}_{2}}\left[\mathrm{O}_{2}\right][\mathrm{X}]=k_{\mathrm{O}_{2}}^{*}[\mathrm{X}] \\
-\frac{\mathrm{d}[\mathrm{X}]}{\mathrm{d} t}=k_{\mathrm{T}}[\mathrm{X}] \\
-\frac{\mathrm{d}[\mathrm{X}]}{\mathrm{d} t}=k_{\mathrm{P}}[h \nu][\mathrm{X}]=k_{\mathrm{P}}^{*}[\mathrm{X}]
\end{gathered}
$$

Eqn (1) and (3) involve constant concentrations of oxygen and light, respectively, and are therefore pseudo-first order reactions, with effective rate constants $k_{\mathrm{O}_{2}}^{*}=k_{\mathrm{O}_{2}}\left[\mathrm{O}_{2}\right]$ and $k_{\mathrm{P}}^{*}=k_{\mathrm{P}}[h \nu]$. With these assumptions we can integrate the three independent reactions (R1)-(R3) to give

$$
\begin{gathered}
{[\mathrm{X}]=[\mathrm{X}]_{0} \exp \left(-k_{\mathrm{O}_{2}}^{*} t\right)} \\
{[\mathrm{X}]=[\mathrm{X}]_{0} \exp \left(-k_{\mathrm{T}} t\right)} \\
{[\mathrm{X}]=[\mathrm{X}]_{0} \exp \left(-k_{\mathrm{P}}^{*} t\right)}
\end{gathered}
$$

Below we will obtain $[\mathrm{X}]$ as a function of time and temperature and fit to eqn (4)-(6) to obtain rate constants and activation energies for these reactions. The concentration of the product formation is governed by the rate equations:

$$
\frac{\mathrm{d}\left[\mathrm{P}_{\mathrm{O}_{2}}, \mathrm{P}_{\mathrm{T}}, \mathrm{P}_{\mathrm{P}}\right]}{\mathrm{d} t}=k_{\mathrm{O}_{2}, \mathrm{~T}, \mathrm{P}}^{*}[\mathrm{X}]-k_{\text {Product }}[\text { Product }]
$$

This can be readily solved assuming that the initial concentration of the product is zero: ${ }^{7}$

$$
\left[\mathrm{P}_{\mathrm{O}_{2}}, \mathrm{P}_{\mathrm{T}}, \mathrm{P}_{\mathrm{P}}\right]=\frac{k_{\mathrm{O}_{2}, \mathrm{~T}, \mathrm{P}}^{*}[\mathrm{X}]_{0}}{k_{\text {Product }}-k_{\mathrm{O}_{2}, \mathrm{~T}, \mathrm{P}}^{*}}\left(\exp \left(-k_{\mathrm{O}_{2}, \mathrm{~T}, \mathrm{P}}^{*} t\right)-\exp \left(-k_{\text {Product }} t\right)\right)
$$

If the product does not degrade further, then $k_{\text {Product }}=0$ and eqn (8) is simply

$$
\left[\mathrm{P}_{\mathrm{O}_{2}}, \mathrm{P}_{\mathrm{T}}, \mathrm{P}_{\mathrm{P}}\right]=[\mathrm{X}]_{0}\left(1-\exp \left(-k_{\mathrm{O}_{2}, \mathrm{~T}, \mathrm{P}}^{*} t\right)\right)
$$

\section{Results and discussion}

\section{Oxidative aging}

When the SNO+ scintillator replica was heated while being exposed to air, the solution transformed from a colorless transparent liquid to a black viscous solution containing solid particulates (Fig. 3). As the sample was oxidized, its fluorescence also decreased substantially.

In Fig. 4 we show the normalized scores of the three components F1, F2 and F3, which correlate to the concentration of the fluorophores PPO, bis-MSB and what appears to be a product of 


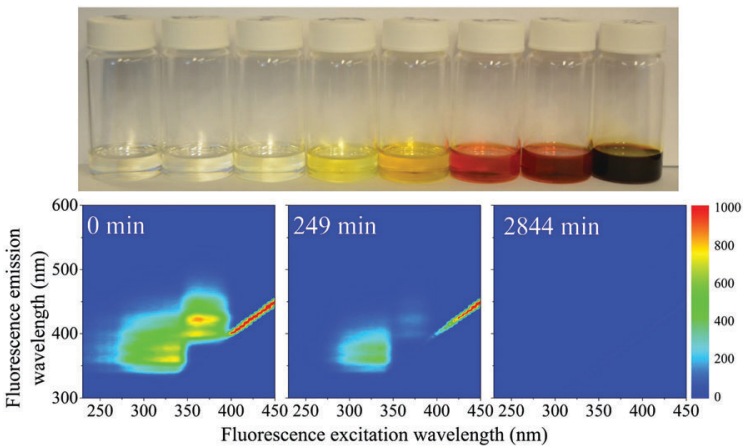

Fig. 3 Top: Photo of the SNO+ scintillator replica after aging under a constant stream of breathing grade air at $426 \mathrm{~K}$. From left to right are degradation times of $0,10,67,249,549,1320,1567,2844$ min. Below: Three EEM spectra of selected samples. The fluorescence vanishes in under $3000 \mathrm{~min}$.

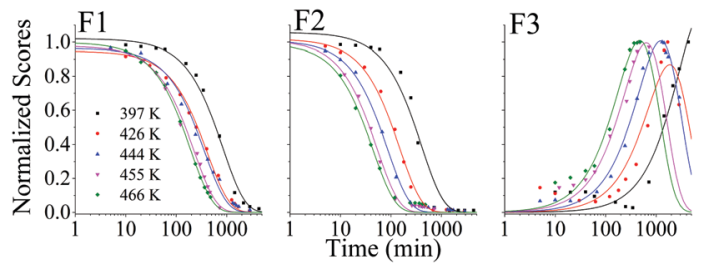

Fig. 4 Normalized PARAFAC scores of the three fluorescent components, F1, F2 and F3 of Fig. 2, after aging the scintillator liquid in breathing grade air. The lines for F1 and F2 are single exponential decay curves obtained by fitting to eqn (4). The solid lines through the F3 data represent a fit to eqn (8).

bis-MSB degradation. As expected, at higher temperatures the sample degrades more quickly. F3 is likely a dimer or oligomer of bis-MSB due to its red shifted spectra. In some experiments it is evident that the scores of $\mathrm{F} 3$ increase and then subsequently decay as the scintillation solution is aged, indicating that this primary oxidation product further decomposes.

The rate constants $k_{\mathrm{O}_{2}}^{*}(\mathrm{X})$ were obtained by fitting to eqn (4) and (8), and are given in Table 1. Activation energies associated with the pseudo-first-order rate constants are obtained from an Arrhenius plot. For the thermal degradation of F1 (PPO) and F2 (bis-MSB) in the presence of oxygen the activation energies are found to be $32.5 \pm 3.7 \mathrm{~kJ} \mathrm{~mol}^{-1}$ and $48.0 \pm 2.7 \mathrm{~kJ} \mathrm{~mol}^{-1}$, respectively (Fig. 5(a)). With these activation energies, the rate constants, $k_{\mathrm{O}_{2}}^{*}(\mathrm{X})$, at room temperature $(298 \mathrm{~K})$ and the temperature of the $\mathrm{SNO}+$ experiment $(285 \mathrm{~K})$ can be determined

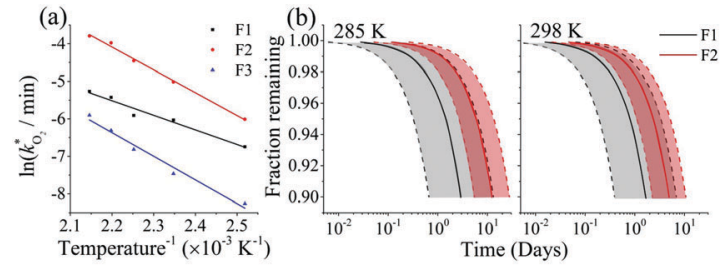

Fig. 5 (a) Arrhenius plot of the rate constants, $k_{\mathrm{O}_{2}}^{*}$, obtained from the fits in Fig. 4. The slopes give the activation energies of F1 (PPO) and F2 (bis-MSB) as $32.4 \pm 3.5 \mathrm{~kJ} \mathrm{~mol}^{-1}$ and $50.5 \pm 1.9 \mathrm{~kJ} \mathrm{~mol}^{-1}$ respectively. The slope of $\mathrm{F} 3$ formation gives an activation energy of $52.4 \pm 4.4 \mathrm{~kJ} \mathrm{~mol}^{-1}$ similar to the decay rate of bis-MSB. (b) The fraction remaining of F1 (black) and F2 (red) at $285 \mathrm{~K}$ and $298 \mathrm{~K}$. The shaded area between the dashed lines represent the upper and lower boundary using one standard deviation of the activation energy. From these activation energies, rate constants were obtained by extrapolation to room temperature ( $298 \mathrm{~K})$ and the temperature of the $\mathrm{SNO}+$ experiment (285 K).

by extrapolation (Table 1). These rates were then used to determine the fraction of F1 (PPO) and F2 (bis-MSB) remaining. Fig. 5(b) shows that in the presence of oxygen, $\mathrm{PPO}$ in the $\mathrm{SNO}+$ scintillation replica degrades to $90 \%(99 \%)$ in only about $3(0.3)$ days at $285 \mathrm{~K}$. Once bis-MSB and PPO are degraded to less than $99 \%$ of their original concentration the scintillator function is affected. Oxygen at ambient concentration (22\% in air) will degrade the scintillator liquid within a few days and must therefore be removed to ensure its longevity. By reducing the oxygen concentration the pseudo-first order rate constant, $k_{\mathrm{O}_{2}}^{*}=k_{\mathrm{O}_{2}}\left[\mathrm{O}_{2}\right]$, is correspondingly reduced and the lifetime can be extended. An oxygen partial pressure in the headspace never exceeding $24 \mathrm{ppm}_{\mathrm{v}}\left(100 \mathrm{ppm}_{\mathrm{v}}\right)$ would reduce the two rate constants to $k_{\mathrm{O}_{2}}^{*}=2.7 \times 10^{-9} \mathrm{~min}^{-1}$ and thus allow the fluorescence of PPO (bis-MSB) to remain within $99 \%$ of its initial value over the anticipated 7 year lifespan.

One may now estimate the maximal permissible concentration of dissolved $\mathrm{O}_{2}$ in the scintillator cocktail. The solubility of $\mathrm{O}_{2}$ in LAB is not given in the literature. However, Battino et al. have performed a comprehensive investigation of the solubility of $\mathrm{O}_{2}$ in a number of related solvents. ${ }^{23,24}$ The solubility of oxygen in LAB may be approximated with that of long chain hydrocarbons such $n$-decane or hexadecane, and the solubility in aromatic hydrocarbons such as butylbenzene. The dissolved mole fraction of $\mathrm{O}_{2}$ in $n$-decane, hexadecane and butylbenzene are $22 \times 10^{-4}, 24 \times 10^{-4}$ and $15 \times 10^{-4}$, respectively, at an oxygen pressure of $101.35 \mathrm{kPa}^{23,24}$ Thus it is expected that in our experiment using a partial $\mathrm{O}_{2}$ pressure of $0.22 \times 101.35 \mathrm{kPa} \mathrm{O}_{2}$ the dissolved mole fraction of $\mathrm{O}_{2}$ in LAB is about 3.2-5.3 $\times 10^{-4}$.

Table 1 Rate constants for the oxidative decay of F1 (PPO) and F2 (bis-MSB) and the rise of F3 (bis-MSB product) in the SNO+ replica scintillation solution obtained by fitting the data shown in Fig. 4 to eqn (4) using $\left[\mathrm{O}_{2}\right]=0.22$. The columns marked with \# give predicted rate constants obtained using the activation energies which were determined from the linear fit in Fig. $5 \mathrm{a}$

\begin{tabular}{|c|c|c|c|c|c|c|c|c|c|}
\hline & \multicolumn{7}{|c|}{ Temperature (K) } & \multirow[b]{2}{*}{$E_{\mathrm{a}}\left[\mathrm{kJ} \mathrm{mol}^{-1}\right]$} & \multirow[b]{2}{*}{$A\left[\mathrm{~min}^{-1}\right]$} \\
\hline & $285^{\#}$ & $298^{\#}$ & 397 & 426 & 444 & 455 & 466 & & \\
\hline$k_{\mathrm{O}_{2}}^{*}(\mathrm{~F} 1)\left[\times 10^{-3} \mathrm{~min}^{-1}\right]$ & 0.0248 & 0.0446 & 1.18 & 2.40 & 2.72 & 4.40 & 5.17 & $32.4(3.5)$ & $21(21)$ \\
\hline$k_{\mathrm{O}_{2}}^{*}(\mathrm{~F} 3)\left[\times 10^{-3} \mathrm{~min}^{-1}\right]$ & 0.000457 & 0.00118 & 0.259 & 0.574 & 1.10 & 1.82 & 2.73 & $52.4(4.4)$ & $2000(2000)$ \\
\hline
\end{tabular}


To protect the scintillator liquid against oxidation the headspace oxygen concentration should not exceed a partial pressure of $2.4 \mathrm{~Pa}\left(24 \mathrm{ppm}_{\mathrm{v}}\right)$ which corresponds to an estimated dissolved $\mathrm{O}_{2}$ mole fraction of 3.5-5.8 $\times 10^{-8}\left(4.3-7.1 \mathrm{ppb}_{\mathrm{w}}\right)$, if the gas in the headspace was completely equilibrated with the liquid.

In addition, the formation of colored oxidation products poses additional limits on the oxygen concentration as is shown in the ESI. $\dagger$ Absorption spectroscopy was used to determine the rate of formation of colored products, but since several products contribute to the spectra and these oxidative reactions occur through several intermediates, it is difficult to unambiguously determine rate constants and activation energies. Even though we were not able to quantitatively establish an upper allowable oxygen concentration, the rate constants for the oxidation product formation obtained through absorption measurements were consistent with those obtained by measuring the degradation of the reactants through fluorescence.

\section{Thermal aging under inert conditions}

The fluorescence EEM spectra of the thermally aged scintillation replica under inert, oxygen-free, conditions are given in Fig. 6. Even when kept at the highest temperature of $513 \mathrm{~K}$, the sample retains its original fluorescence intensity for nearly two months. The normalized scores obtained by PARAFAC analysis are given in Fig. 7(a) and are fit to an exponential decay function, to model a first-order reaction, eqn (5). As the scores do not decrease enough to generate a reliable exponential decay, a worst case scenario was implemented, by adding an extra data point located at $50 \%$ of the normalized score at the next, future, time interval (200 days). This time interval was determined using the same fit to the exponential decay function, separately for both components.

The rate constants obtained in the fit of Fig. 7(a) are given in Table 2. The Gibbs free energies of activation, $\Delta G^{\ddagger}$, were obtained using the Eyring-Polanyi equation below and are also given in Table 2 .

$$
k_{\mathrm{T}}=\frac{k_{\mathrm{B}} T}{h} \exp \left(-\frac{\Delta G^{\ddagger}}{R T}\right)
$$

The rate at $285 \mathrm{~K}$ - the temperature of the $\mathrm{SNO}+$ experimentcould then be determined by extrapolation. Using eqn (10), the fraction of F1 (PPO) and F2 (bis-MSB) remaining in the SNO+ scintillation replica was determined and is shown in Fig. 7(b). As observed, even in a worst case scenario, the SNO+ scintillation

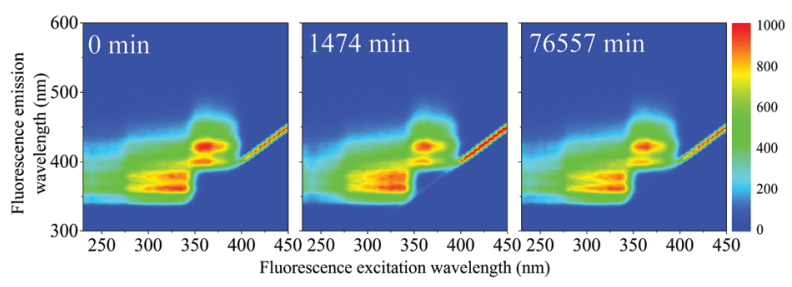

Fig. 6 EEM spectra of samples obtained after heating to $513 \mathrm{~K}$ under inert conditions. The fluorescence remains strong even after aging for nearly 2 months.
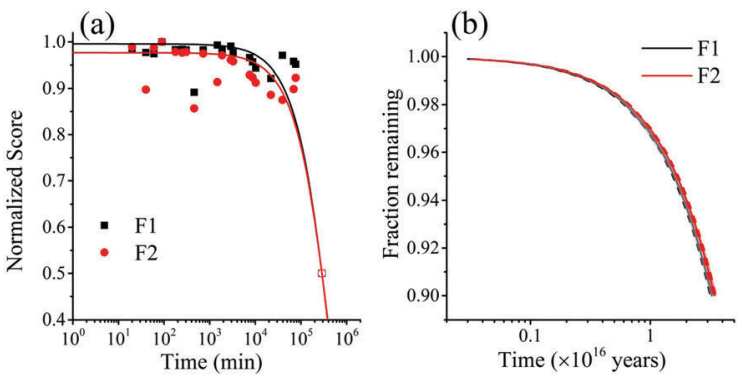

Fig. 7 (a) Normalized PARAFAC scores of components F1 (PPO) and F2(bis-MSB) obtained by analysis of the EEM spectra after heating to $513 \mathrm{~K}$. Component F3 was excluded as its contribution was negligible. The colored lines represent single exponential decay fits through the scores using eqn (5). The hollow data points at 50\% were added to help the exponential fit and are used as a worst case scenario. (b) Fraction remaining of F1 (black) and F2 (red) at $285 \mathrm{~K}$ if the scintillator is kept under inert conditions. The remaining fraction was obtained by calculating the Gibbs free energy of activation, $\Delta G^{\ddagger}$, obtained from the rates in Fig. 10(a) with eqn (10). The shaded areas between the dashed lines represent one standard deviation of the rate at $285 \mathrm{~K}$.

Table 2 Rate constants for the thermal decay of F1 (PPO) and F2 (bis$\mathrm{MSB}$ ) in the $\mathrm{SNO}+$ replica scintillation solution in the absence of oxygen obtained by fitting the data shown in Fig. 7(a) to eqn (5). The column marked with \# gives predicted rate constants obtained using the listed value for $\Delta G^{*}$

\begin{tabular}{llll}
\hline & \multicolumn{2}{l}{ Temperature (K) } & \\
\cline { 2 - 3 } & $285^{\#}$ & 513 & $\Delta G^{\ddagger}\left(\mathrm{kJ} \mathrm{mol}^{-1}\right)$ \\
\hline$k_{\mathrm{T}}(\mathrm{F} 1)\left(\mathrm{s}^{-1}\right)$ & $1.03(0.03) \times 10^{-24}$ & $2.39(0.04) \times 10^{-6}$ & $200.7(0.1)$ \\
$k_{\mathrm{T}}(\mathrm{F} 2)\left(\mathrm{s}^{-1}\right)$ & $0.98(0.03) \times 10^{-24}$ & $2.32(0.04) \times 10^{-6}$ & $200.8(0.1)$
\end{tabular}

replica is extremely stable when kept under inert conditions. It will take approximately $3.4 \times 10^{15}\left(3.2 \times 10^{14}\right)$ years for the bisMSB concentration to degrade to $90 \%$ (99\%) of its original concentration.

As expected, the absorption (not shown) does not noticably change as the sample is aged under inert conditions and, similar to the EEM spectra, the absorption spectra at 0 and 76557 min remain nearly identical.

\section{Photochemical aging}

When exposed to UV light at $365 \mathrm{~nm}$, the fluorescence of the $\mathrm{SNO}+$ scintillation solution replica rapidly diminishes as shown in Fig. 8. This is clearly visible as the blue fluorescence decreases after samples were illuminated by UV radiation. The normalized scores obtained by PARAFAC analysis are given in Fig. 9 and are fit to an exponential decay. Inner filter effects are apparent through the slight increase of the PPO fluorescence until bis-MSB has degraded. Thus, only the scores of PPO after the maximum were used to determine the rate constants from the fit to eqn (6).

Dividing the pseudo-first order rate constants, $k_{\mathrm{P}}^{*}=k_{\mathrm{P}}[h \nu]$ by the photon concentration of $[h \nu]=4.25 \pm 0.17 \times 10^{-7}$ photon $\mathrm{mol} \mathrm{L}^{-1}$ allows us to obtain the second order rate constants, $k_{\mathrm{P}}$ (Table 3 ). As with the thermally aged samples, the Gibbs free energies of activation, $\Delta G^{\ddagger}$, of the photoreaction were obtained using eqn (10). 


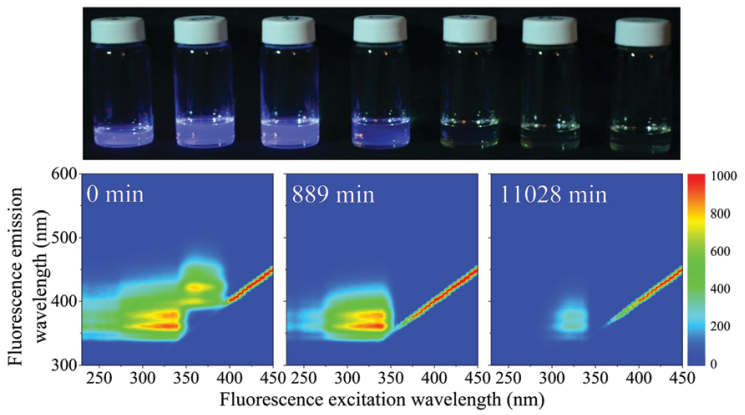

Fig. 8 Top shows a photo of the photodegraded $\mathrm{SNO}+$ scintillation solution after irradiation by $365 \mathrm{~nm}$ UV light. From left to right show samples taken at 0,20,123, 449, 1329, 2759, $7049 \mathrm{~min}$. Below shows three selected EEM spectra of the same samples. The fluorescence intensity decreases as the sample is exposed to UV light.

With $\Delta G^{\ddagger}$, the rate of degradation at the SNO+ experiment conditions, $285 \mathrm{~K}$, was determined by extrapolation. The photon concentrations required to degrade the $\mathrm{SNO}+$ scintillation solution replica to $99 \%$ and $90 \%$ of the fluorophores original concentration are given in Fig. 9(b). Fig. 9 shows that
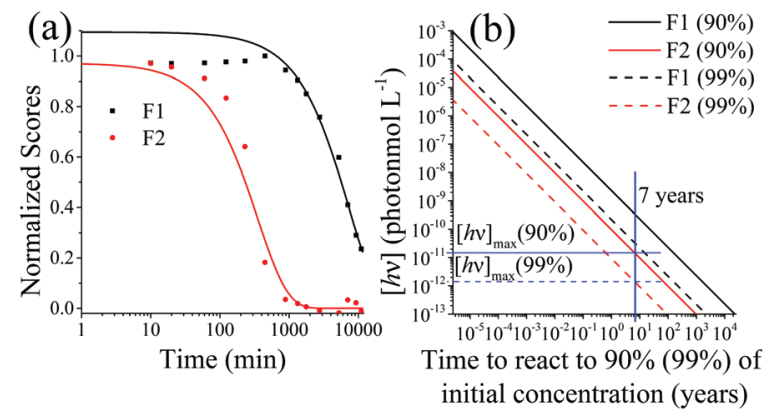

Fig. 9 (a) Normalized scores of F1 (PPO) and F2 (bis-MSB) of the photodegraded samples obtained using PARAFAC analysis. The lines represent single exponential decay processes and are fit to eqn (6). The fit for F1 was only conducted on the decreasing portion of the data, due to inner filter effects. (b) Concentration of photons to degrade the SNO+ scintillation replica to $90 \%$ (Solid lines) or $99 \%$ (dashed lines) of its original concentration at $285 \mathrm{~K}$. The solid blue line represents the 7 years of the $\mathrm{SNO}+$ experiment and the horizontal blue lines show the maximum concentration of allowed photons that the solution can be constantly exposed to in order to ensure a $90 \%$ or $99 \%$ effective solution. These were obtained by calculating the Gibbs free energy of activation, $\Delta G^{\ddagger}$.
bis-MSB is much more sensitive than PPO to photochemical decomposition under UV-radiation. The maximum concentration of UV photons at $365 \mathrm{~nm}$ that can irradiate the scintillation solution to remain $90 \%$ (99\%) fluorescent over 7 years was determined to be $1.38 \pm 0.09 \times 10^{-11}$ photon $\mathrm{mol} \mathrm{L}^{-1}$ $\left(1.32 \pm 0.08 \times 10^{-12}\right.$ photon $\left.\mathrm{mol} \mathrm{L}^{-1}\right)$.

Absorption spectra of the UV aged samples, Fig. 10(a), show that the absorption also decreases with increased exposure. PARAFAC analysis of the 17 photodegraded sample absorption spectra led to a two component fit which is given in Fig. 10(b). Component AP1 resembles the absorption spectrum of PPO, Fig. S1(b) in the ESI $\dagger$, and its scores decrease with a first order decay. A fit to eqn (6) with $[h \nu]=4.25 \pm 0.17 \times 10^{-7}$ photon $\mathrm{mol} \mathrm{L}^{-1}$ gives $k_{\mathrm{P}}(\mathrm{AP} 1)=19.5(1.1) \mathrm{L}$ photon $\mathrm{mol}^{-1} \mathrm{M}^{-1} \mathrm{~s}^{-1}$ at $298 \mathrm{~K}$. Although the absorption spectrum matches that of PPO this decay rate does not match the decay constant of the fluorescence component F1(PPO), $k_{\mathrm{P}}(\mathrm{F} 1)=5.41(0.18) \mathrm{L}$ photon $\mathrm{mol}^{-1} \mathrm{M}^{-1} \mathrm{~s}^{-1}$. AP1 must then be attributed to both the degradation of PPO and another process that occurs much faster-the degradation of bis-MSB as a possible example.

The second component, AP2, which has $\lambda_{\max } \approx 280 \mathrm{~nm}$ increases as the sample is degraded and a fit to eqn (9) gives $k_{\mathrm{P}}(\mathrm{AP} 2)=5.8$ (1.2) $\mathrm{L}$ photon $\mathrm{mol}^{-1} \mathrm{M}^{-1} \mathrm{~s}^{-1}$ at $298 \mathrm{~K}$. This matches the rate of the degradation of $\mathrm{F} 1$ (PPO) from the fluorescence data (Table 3) and it is therefore likely that component AP2 is a primary $\mathrm{PPO}$ photodegradation product. Given that the absorption peak of AP2 at $280 \mathrm{~nm}$ (Fig. 10(b)) is similar to those of phenyl groups it is plausible that PPO photochemically degrades by breaking its conjugation. The above paragraph illustrates that the interpretation of absorption

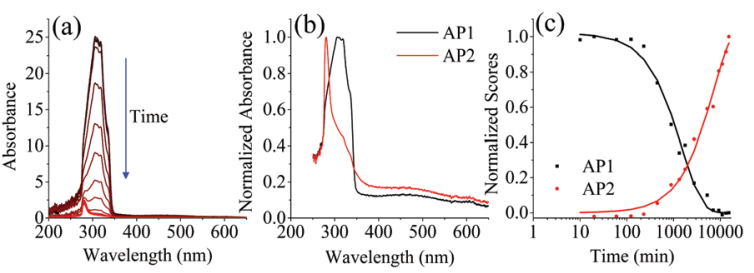

Fig. 10 (a) Absorption spectra of the photo degraded samples. As the samples are exposed to UV light the absorbance decreases. (b) The two components determined using PARAFAC (AP1, AP2) on the 17 UV degraded samples. (c) Shows the normalized absorbance scores of both components shown in b. The lines represent fits to the data using eqn (6) and (9).

Table 3 Rate constants for the decay of F1 (PPO) and F2 (bis-MSB), and AP1 and the rise of AP2 in the SNO+ replica scintillation solution obtained by fitting the data shown in Fig. $9(a)$ and 10 (c) to eqn (6) and (9). The second order rate constant, $k_{\mathrm{P}}$, was obtained using $k_{\mathrm{P}}=k_{\mathrm{P}}^{*} /[h \nu]$ where $[h \nu]=4.25 \pm 0.17 \times 10^{-7}$ photon $\mathrm{mol} \mathrm{L}^{-1}$. The column marked with \# gives predicted rate constants obtained using the listed value for $\Delta G^{\sharp}$. The values for $[h \nu]_{\max }$ represent the maximum concentration of UV photons allowed to ensure the solution remains $90 \%$ and $99 \%$ fluorescent over the 7 years. These values are shown graphically in Fig. 9(b) for F2

\begin{tabular}{|c|c|c|c|c|c|}
\hline & \multicolumn{2}{|c|}{ Temperature (K) } & $\Delta G^{\ddagger}\left(\mathrm{kJ} \mathrm{mol}{ }^{-1}\right)$ & \multicolumn{2}{|c|}{$\left.\underline{[h \nu]_{\max }\left(\times 10^{-10} \text { photon mol L }\right.}{ }^{-1}\right)$} \\
\hline$k_{\mathrm{P}}(\mathrm{F} 1)\left(\mathrm{L}\right.$ photon $\left.\mathrm{mol}^{-1} \mathrm{M}^{-1} \mathrm{~s}^{-1}\right)$ & $1.458(0.052)$ & $5.41(0.18)$ & $68.84(0.08)$ & $3.27(0.11)$ & $0.312(0.011)$ \\
\hline
\end{tabular}


spectra to extract the reaction kinetics of a complex degradation process is neither simple nor unambiguous.

\section{Conclusions}

Excitation-emission matrix spectroscopy was combined with parallel factor analysis to determine the stability of the SNO+ scintillation solution under oxidative, thermal, and photochemical stress and to specify the necessary conditions needed to ensure its function for the anticipated 7 year duration of the $\mathrm{SNO}+$ experiment. ${ }^{25}$ It was determined that the presence of $\mathrm{O}_{2}$ in the scintillation flask or headspace is very detrimental to the SNO+ experiment and the amount of $\mathrm{O}_{2}$ must be below a partial pressure in the headspace of $2.4 \mathrm{~Pa}\left(24 \mathrm{ppm}_{\mathrm{v}}\right)$ corresponding to an approximate dissolved mole fraction of 3.5 to $5.8 \times 10^{-8}$ (4.3-7.1 $\mathrm{ppb}_{\mathrm{w}}$ ). This condition must be fulfilled to ensure that the solution would remain $90 \%$ fluorescent after 7 years - the anticipated duration of the SNO+ experiment. The absorption data shown in the ESI $\dagger$ are consistent with these values.

We note that oxygen can be removed quite effectively from water and other solvents by bubbling purified nitrogen gas through solution. It was shown previously that about $500 \mathrm{ppb}_{\mathrm{w}}$ of $\mathrm{O}_{2}$ (mole fraction of $4.1 \times 10^{-6}$ ) remain dissolved in water after purging with nitrogen. ${ }^{26}$ This concentration would be equilibrated to a concentration of $2700 \mathrm{ppm}_{\mathrm{v}}$ in the headspace. Simple purging of the scintillator liquid with $\mathrm{N}_{2}$ may therefore not be sufficient to ensure the longevity of the solution, if the scintillator was allowed to equilibrate with an infinitely large headspace. In the SNO+ experiment, however, the headspace volume is very small compared to the volume of the liquid and the supply of $\mathrm{O}_{2}$ is limited. In both estimates of the maximum allowable $\mathrm{O}_{2}$ concentration it was assumed that the concentration of $\mathrm{O}_{2}$ in the headspace was constant. If the SNO+flask was kept airtight and the headspace very small, the oxidation process would terminate as soon as the $\mathrm{O}_{2}$ supply is depleted. Trivially, the depletion reaction will stop after all $\mathrm{O}_{2}$ has reacted with the large excess of PPO.

It is also apparent that after purging the scintillator with purified $\mathrm{N}_{2}$ and keeping the SNO+ scintillation solution under inert gas the solution is expected to remain useable for much longer than 7 years. When handling the SNO+ scintillator liquid, e.g. when filling tanker trucks, storage vessels, pipes, and, of course, the SNO+ detector, it is therefore important to first purge the empty container with an inert gas before filling it with scintillator liquid.

In addition, adding an oxygen quencher such as 1,4-diazabicyclo[2.2.2] octane (DABCO) may improve the lifetime of the fluorescent dyes and increase the $\mathrm{SNO}+$ scintillation solutions lifetime. ${ }^{27}$ There are inherent risks in adding even more compounds to the scintillator mixture, however, as they may also contaminate the liquid.

We also showed that UV radiation degrades the SNO+ scintillation solution and measures must be taken to ensure that the average concentration of photons in the flask remains lower than $1.38 \pm 0.09 \times 10^{-11}$ photon $\mathrm{mol} \mathrm{L}^{-1}$. As a comparison the photon flux of a UV curing lamp is about $4.25 \pm 0.17 \times 10^{-7}$ photon $\mathrm{mol} \mathrm{L}^{-1}$. While the SNO+ scintillator will be kept in the dark during the experiment, precautions are also needed when preparing the solution.

The degradation studies presented in this paper on the $\mathrm{SNO}+$ liquid scintillator can be used as a guide for other liquid scintillator systems. Many scintillators contain similar fluorophores such as oxazoles or conjugated $\pi$-systems and would be expected to degrade in similar reactions. While their rate constants with regards to their reactions with oxygen and light are different, the techniques described in this article are readily transferrable to future studies on the stability of liquid scintillators.

Finally, in the present study the SNO+ scintillator replica did not contain tellurium, but in the second phase of the SNOexperiment ${ }^{130} \mathrm{Te}$ will have to be dissolved either as part of an inverted micellar solution or through forming a soluble complex. It is then quite likely that the oxidative stability of the cocktail will be affected and a second study is required.

The main advantage of combining EEM fluorescence spectra with PARAFAC analysis is its ability in extracting kinetic information. Although determining the optical performance and stability of the scintillator using spectroscopy is not unique, the approach presented in this work may be the first spectroscopic analysis where the performance of a scintillator (fluorescence, absorption) is determined in a multi-parameter system (temperature, UV exposure, $\mathrm{O}_{2}$ exposure, time).

It is likely that this combination of methods is also suitable to study other mixtures of fluorescent species, such as the degradation of foodstuff, environmental samples, lubricants, and other industrial liquids. ${ }^{9}$

\section{Acknowledgements}

The authors thank Igor Kozin for lending the absorption spectrometer to us, Cathleen Crudden for use of her recrystallization oven, and Weatherford International for lending us the Fluorescence EEM spectrometer. We also acknowledge funding by the Natural Sciences and Engineering Research Council (NSERC) of Canada.

\section{References}

1 J. Boger, R. L. Hahn and J. K. Rowley, et al., (SNO Collaboration), Nucl. Instrum. Methods Phys. Res., Sect. A, 2000, 449, 172-207.

2 M. C. Chen, Nucl. Phys. B, Proc. Suppl., 2005, 145, 65-68.

3 F. P. An, Q. An and J. Z. Bai, et al., (Daya Bay Collaboration), Nucl. Instrum. Methods Phys. Res., Sect. A, 2012, 685, 78-97.

4 J. S. Park, J. Lee and I. S. Yeo, et al., (RENO Collaboration), Nucl. Instrum. Methods Phys. Res., Sect. A, 2013, 707, 45-53.

5 D. Airado-Rodríguez, I. Durán-Merás, T. Galeano-Díaz and J. P. Wold, J. Food Compos. Anal., 2011, 24, 257-264.

6 P. G. Coble, Mar. Chem., 1996, 51, 325-346.

7 H. Omrani, A. E. Dudelzak, B. P. Hollebone and H. P. Loock, Anal. Chim. Acta, 2014, 811, 1-12. 
8 H. Omrani, J. A. Barnes, A. E. Dudelzak, H.-P. Loock and H. Waechter, Analyst, 2012, 137(2782), 8.

9 N. L. P. Andrews, J. Z. Fan, H. Omrani, A. Dudelzak and H.-P. Loock, Tribol. Int., 2016, 94, 279-287.

10 K. R. Murphy, C. A. Stedmon, D. Graeber and R. Bro, Anal. Methods, 2013, 5(23), 6557-6566.

11 R. Bro and H. A. L. Kiers, J. Chemom., 2003, 17(5), 274-286.

12 D. T. Mahin, Int. J. Appl. Radiat. Isot., 1966, 17, 185-191.

13 R. W. Pringle, L. D. Black, B. L. Funt and S. Sobering, Phys. Rev., 1953, 92, 1582-1583.

14 C. G. Hatchard and C. A. Parker, Proc. R. Soc. London, Ser. A, 1956, 235, 518-536.

15 H.-L. Xiao, X.-B. Li, D. Zheng, J. Cao, L.-J. Wen and N.-Y. Wang, Chin. Phys. C, 2010, 34, 1724.

16 N. L. P. Andrews, A. G. MacLean, J. E. Saunders, J. A. Barnes, H. P. Loock, M. Saad, C. L. Jia, K. Ramaswamy and L. R. Chen, Opt. Express, 2014, 22(16), 19337.

17 J. McMurry, Organic Chemistry (Brooks/Cole Publishing Company), Cornell University, 2012, pp. 1215-1219.
18 T. J. H. M. Cuppen and W. H. Laarhoven, J. Am. Chem. Soc., 1972, 94, 5914-5915.

19 F. B. Mallory and C. W. Mallory, Organic Reactions, John Wiley \& Sons, Inc., 2004.

20 M. E. Ackerman, G. H. Daub, F. N. Hayes and H. A. Mackay, in Organic Scintillators and Scintillation Counting, ed. D. Horrocks, Elsevier, 2012, pp. 315-325.

21 C. Geletneky and S. Berger, Eur. J. Org. Chem., 1998, 1625-1627.

22 D. a. Maurizio, Adv. Heterocycl. Chem., Elsevier, 2001, vol. 79, pp. 41-88.

23 R. Battino, T. R. Rettich and T. Tominaga, J. Phys. Chem. Ref. Data, 1983, 12(2), 163-178.

24 H. L. Clever, R. Battino, H. Miyamoto, Y. Yampolski and C. L. Young, J. Phys. Chem. Ref. Data, 2014, 43(3), 033102.

25 S. Biller, presented at the 13th Int. Conf. on Topics in Astroparticle and Underground Physics, 2014, unpublished.

26 I. B. Butler, M. A. A. Schoonen and D. T. Rickard, Talanta, 1994, 41(2), 211-215.

27 C. Schweitzer and R. Schmidt, Chem. Rev., 2003, 103, 1685-1758. 